\title{
Paraesophageal Hernia as a Cause of Chronic Asymptomatic Anemia in a 6 Years Old Boy; Case Report and Review of the Literature
}

\author{
Dimitrios Patoulias*, Maria Kalogirou, Thomas Feidantsis, Ignatios Kallergis, Ioannis Patoulias
}

\begin{abstract}
Esophageal hiatal hernia is defined as the prolapse of one or more intra-abdominal organs through the esophageal hiatus. Four types are identified: type I or sliding hiatal hernia, type II or paraesophageal hernia (PEH), type III or mixed hernia and type IV. Congenital type II esophageal hiatal hernia is caused by a remaining gap after the formation of pleuroperitoneal membrane. We present a case of a six years old boy admitted to our department, appearing with asymptomatic anemia, who was incidentally diagnosed with Type II esophageal hiatal hernia. After diagnostic investigation, the prolapsing stomach pouch was reduced, the hernia sac was excised, the crura of diaphragm were converged and a total fundoplication was performed, via open method. The patient had an uncomplicated postoperative period. We conclude that: 1) esophageal hiatal hernia should be included within diagnostic approach of a child with chronic non-hereditary anemia, 2) after a Type II esophageal hiatal hernia is diagnosed, a hernia repair surgery is indicated in short time, due to the severity of possible complications and 3) through the performance of total fundoplication, it is secured that the subdiaphragmatic abdominal part of esophagus will be retained, preventing the development of post-operative gastroesophageal reflux disease.
\end{abstract}

\section{KEYWORDS}

type II esophageal hiatal hernia; gastric volvulus; child; gastroesophageal reflux disease; fundoplication

\section{AUTHOR AFFILIATIONS}

1st Department of Pediatric Surgery, Aristotle University of Thessaloniki, GH G. Gennimatas, 41 Ethnikis Aminis Street, 54635, Thessaloniki, Greece

*Corresponding author: M. Alexandrou 3B, Peuka, 57010 Thessaloniki, Greece; e-mail: dipatoulias@gmail.com

Received: 30 January 2017

Accepted: 19 April 2017

Published online: 5 October 2017

Acta Medica (Hradec Králové) 2017; 60(2): 76-81

https://doi.org/10.14712/18059694.2017.97

(c) 2017 The Authors. This is an open-access article distributed under the terms of the Creative Commons Attribution License (http://creativecommons.org/licenses/by/4.0), which permits unrestricted use, distribution, and reproduction in any medium, provided the original author and source are credited. 


\section{INTRODUCTION}

Esophageal hiatal hernia is defined as the prolapse of one or more intra-abdominal organs through the esophageal hiatus. Four types are identified: type I or sliding hiatal hernias (85-95\%), type II or paraesophageal hernias $(3.5-5 \%)$, type III or mixed hernias (10\%), and type IV or complex hernias $(0-5 \%)(1,2)$. Based upon the width of esophageal hiatus, two types are identified: wide (diameter $>2 \mathrm{~cm}$ ) and small (diameter $<2 \mathrm{~cm}$ ) (3).

Type II hiatal hernias are divided into congenital and acquired. Congenital type II hiatal hernia is caused by a remaining gap after the fusion of pleuroperitoneal folds and the formation of pleuroperitoneal membrane. During intrauterine life, this gap has a diameter less than $1 \mathrm{~cm}$. Its dimensions increase over time $(4,5)$. Alternatively, the gap is, also, caused by abnormal formation of the lumbar part of diaphragm from mesodermal cells, originating from lumbar spine. Congenital hiatal hernias are more common in girls (Female/Male ratio = 4) (6). Baglaj SM et al. refer to 21 cases of type II hiatal hernias with familial predisposition, 6 of which were cases of twins (7).

Acquired hiatal hernias are more common in children suffering from cerebral paralysis, chronic connective tissue diseases, such as Ehlers-Danlos syndrome and Marfan syndrome, whereas, they can, also, occur as a postoperative complication of anti-reflux surgery, when the crura of diaphragm where, technically, not properly converged $(8,9,10)$.

In type II hiatal hernia, the hernia sac is located in the right side of esophagus - due to insufficient development of the right crus of diaphragm - and inside the sac a part or the entire stomach prolapses (11). In early stages, the gastroesophageal angle remains acute and is located within the abdominal cavity. Owing to negative intrathoracic pressure, the prolapsing stomach pouch, usually, remains within the thorax.

Symptomatology of type II hiatal hernias involves recurrent infections of the lower respiratory tract, atypical symptoms of the gastrointestinal tract, whereas in $8-8.48 \%$ of all cases it may remain asymptomatic $(1,11)$. Karpelowsky JS et al. encountered successfully 59 pediatric patients (median age $=23.4$ months old) with type II hiatal hernia over 42 years (1). The majority of the patients suffered from more than one symptoms: 32 patients (54.24\%) presented with recurrent infections of the lower respiratory tract, $24(40.68 \%)$ with repeated vomiting after meal, 20 (33.9\%) with symptomatic anemia, 18 (30.51\%) with growth delay and $6(10.17 \%)$ with dysphagia. Five out of fifty nine patients (8.48\%) remained asymptomatic, with the diagnosis made incidentally (1).

In this case report we present a rare, atypical and, essentially, subclinical case of Type II hiatal hernia. After comprehensive research of the current, relevant literature, we attempt to answer critical questions, which remain topical.

\section{CASE REPORT}

We present a case of six years old boy, who was admitted to the Emergency Department of the 1st Department of Pedi- atric Surgery, Aristotle University of Thessaloniki, due to incidental ingestion of a radiopaque foreign body 12 hours ago. The little patient had free family and perinatal history. His medical history was remarkable for undiagnosed iron deficiency, resistant to therapy and early satiety after low volume meals.

The child had normal lung sounds and intestinal peristalsis. A posteroanterior neck, chest and abdominal x-ray were conducted, which - except from the radiopaque foreign body in the right lower quadrant of the abdomen showed a radiolucent spherical halo in the lower third of the mediastinum (Fig. 1).

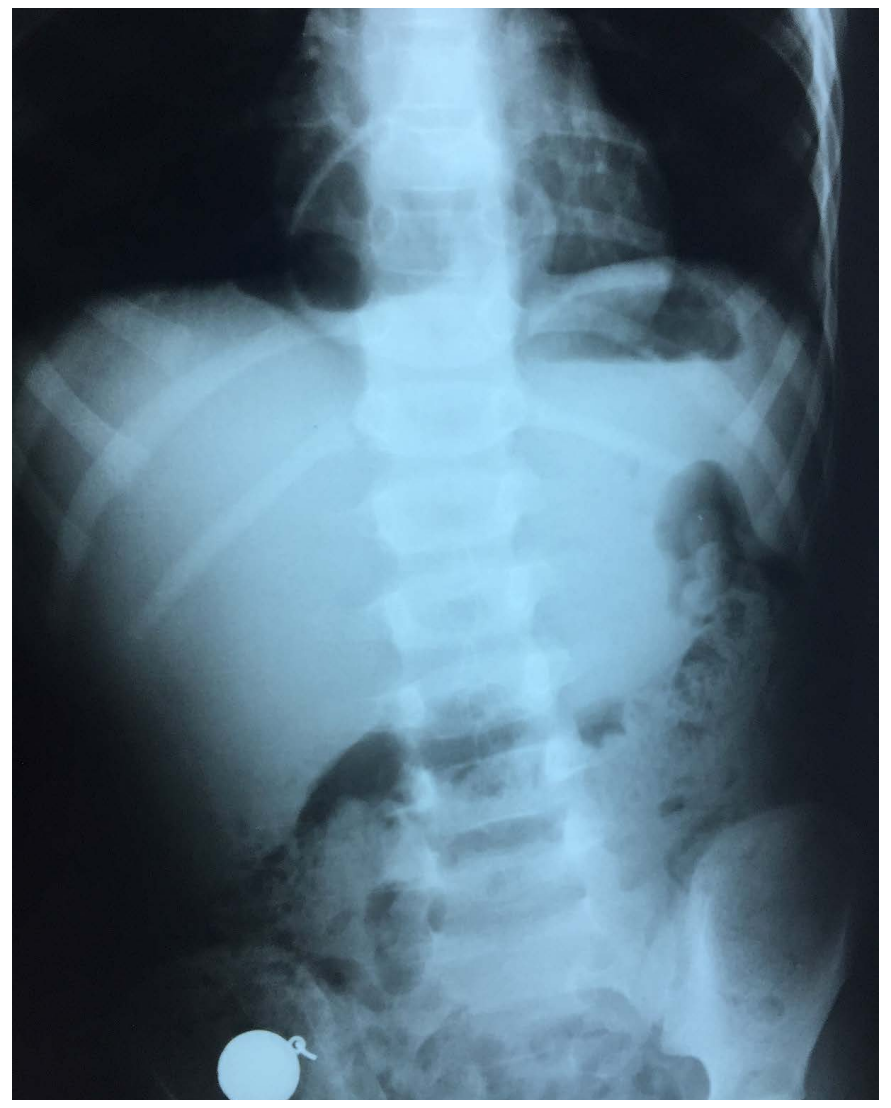

Fig. 1: A radiolucent spherical halo in the lower third of the mediastinum.

Subsequently, a lateral chest $\mathrm{x}$-ray revealed the subvertebral location (in the posterior mediastinum) of this semicircular radiolucent spherical halo (Fig. 2).

Laboratory tests confirmed the iron deficiency, while homozygous or heterozygous hemoglobinopathy were excluded $\left(\mathrm{HbA}_{2}: 2.7 \%, \mathrm{HbF}<0.5 \%\right)$ (Table 1$)$.

Tab. 1: Preoperative values of hematological parameters.

\begin{tabular}{|l|l|}
\hline Parameter & Value \\
\hline $\mathrm{Ht}$ & $39 \%$ \\
\hline $\mathrm{Hb}$ & $11.3 \mathrm{~g} / \mathrm{dl}$ \\
\hline $\mathrm{MCV}$ & $59.3 \mathrm{fl}$ \\
\hline $\mathrm{MCH}$ & $18.3 \mathrm{pg}$ \\
\hline $\mathrm{MCHC}$ & $30.9 \mathrm{~g} / \mathrm{dl}$ \\
\hline RDW & $20.8 \%$ \\
\hline Ferritin & $1.5 \mu \mathrm{g} / \mathrm{dl}$ \\
\hline
\end{tabular}




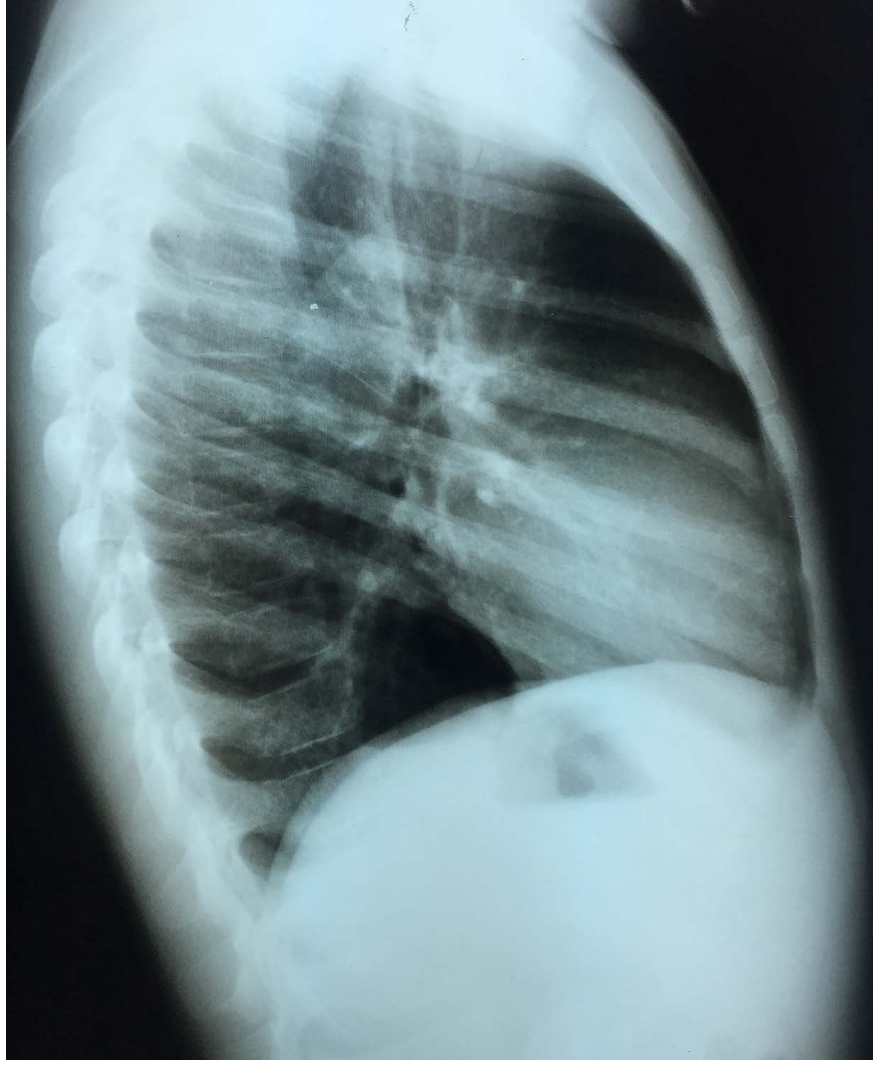

Fig. 2: Subvertebral location (in the posterior mediastinum) of this semicircular radiolucent spherical halo.

Afterwards, barium meal examination was conducted, which confirmed the intra-thoracic location of stomach's fundus and part of stomach's body and revealed a hourglass stenosis in the esophageal hiatus. The gastroesophageal junction appeared to remain within the abdominal cavity. Furthermore, no gastroesophageal reflux or delayed gastric emptying was noticed (Fig. 3).

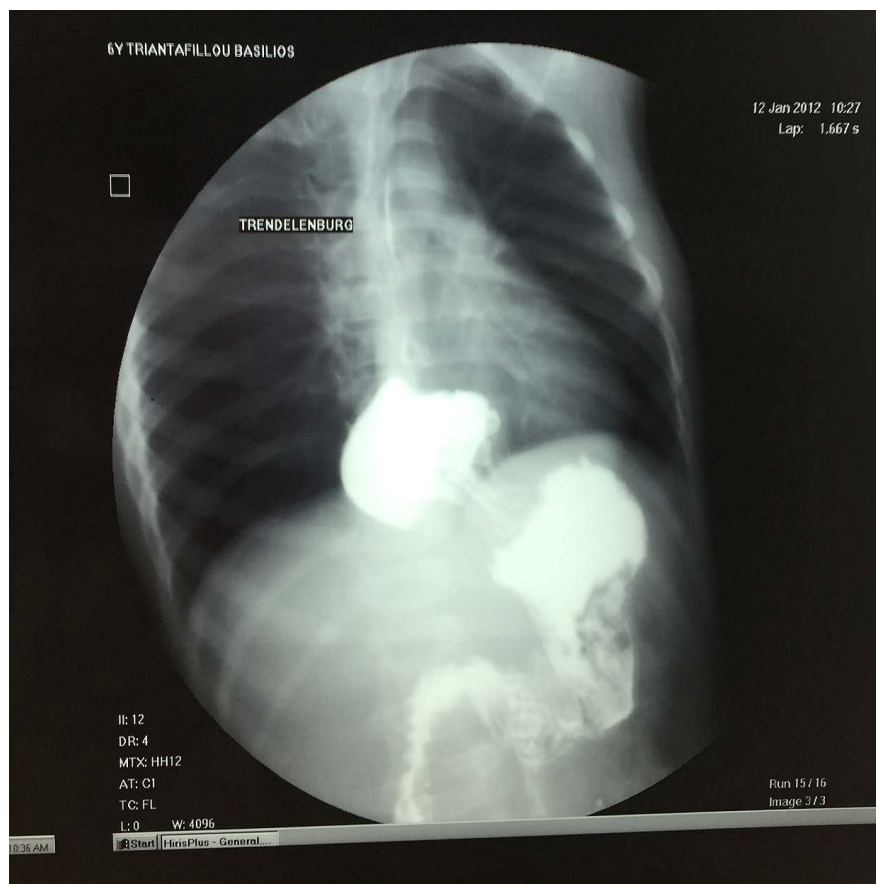

Fig. 3: Intra-thoracic location of stomach's fundus and part of stomach's body with a hourglass stenosis in the esophageal hiatus.
Endoscopy of the upper digestive tract did not show any signs of esophagitis, but revealed an oversize type II hiatal hernia, with a prolapse of the stomach's fundus and part of its body (Fig. 4).

Subsequently, MRI of the chest and upper abdomen revealed the width of the gap $(4 \mathrm{~cm})$, the location of the stomach's fundus and part of its body within the hernia sac and the intra-abdominal location of the gastroesophageal junction (Fig. 5).

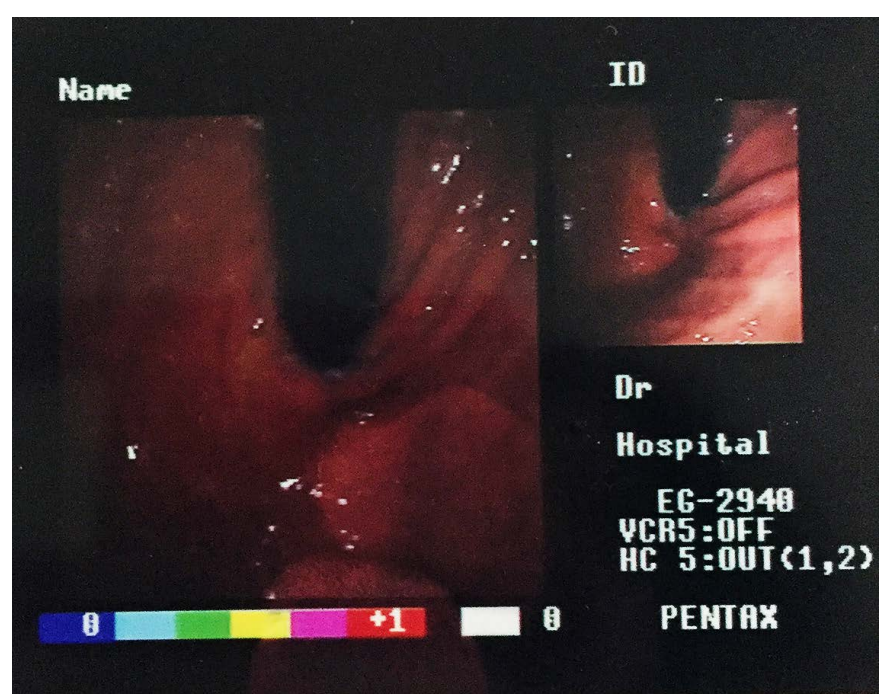

Fig. 4: Notice the wide gap through which the stomach prolapses, in the context of a type II hiatal hernia.

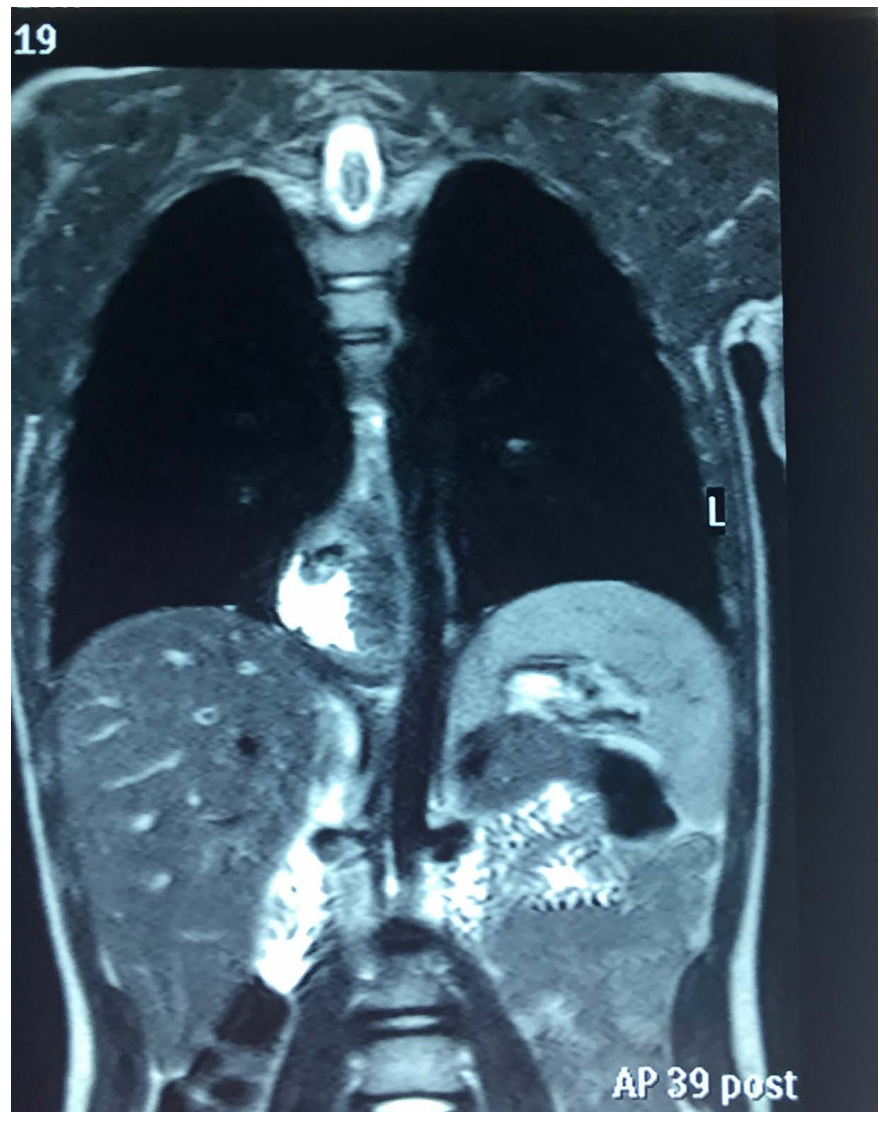

Fig. 5: MRI of the chest and upper abdomen reveals the width of the gap $(4 \mathrm{~cm})$, the location of the stomach's fundus and part of its body within the hernia sac and the intra-abdominal location of the gastroesophageal junction. 
After diagnostic documentation of Type II hiatal hernia, elective surgical repair followed. With the patient under general endotracheal anesthesia and after conduction of a left mini Kocher section, the prolapsed stomach pouch was reduced, the hernia sac and the adhesions of the right crus of diaphragm in the parietal pleura and in the pericardium were excised, the crura of diaphragm were converged with 3 separate Vigryl 2/0 stitches and a total $360^{\circ}$ fundoplication was performed (Nissen's fundoplication).

\section{RESULTS}

The little patient had an early recovery from anesthesia, after surgery. On the second postoperative day, the nasogastric tube was removed and we began gradually oral feeding. On the fifth postoperative day, the patient was discharged home in excellent general condition. The first postoperative clinical evaluation was performed after 3 months, when the excellent general condition of the patient was documented. The quantity of food was, gradually, increased without any episode of gastric dilatation or dysphagia. Blood test revealed an increase in ferritin levels up to $45 \mu \mathrm{g} / \mathrm{dl}$. Also, red blood cell indices significantly improved compared to those postoperatively (MCV: $77.8 \mathrm{fl}$ vs. $59.3, \mathrm{MCH}: 25.7 \mathrm{pg}$ vs. 18.3 and MCHC: $33 \mathrm{~g} / \mathrm{dl}$ vs. 30.9 ).

One year later, the patient remains asymptomatic with normal hematological profile. Barium meal examination was conducted again, and confirmed the intra-abdominal location of the stomach, the acute gastroesophageal angle, no gastroesophageal reflux and a normal gastric emptying (Fig. 6, 7).

\section{DISCUSSION}

Until now, less than 10 case reports of prenatally diagnosed hiatal hernia have been published $(12,13,14)$. When an intra-thoracic cystic mass is found, the differential diagnosis should include diseases, such as hiatal hernia, macrocystic adenomatoid malformation, esophageal duplication cyst, neurenteric cyst and pericardial cyst. Yamamoto $\mathrm{N}$ et al. diagnosed a Type II hiatal hernia in a fetus with asplenia syndrome (absence of spleen, dextrocardia, atrial and ventricular septal defect, pulmonary artery stenosis and malrotation) (14). Possibly, as prenatal evaluation rapidly evolves, there will be more prenatally diagnosed congenital hiatal hernias cases in the future. This possible evolution would change the landscape of diagnostic investigation and repair of hiatal hernias, even in a subclinical stage.

Early symptoms of type II hiatal hernias are not caused by gastroesophageal reflux, as the anti-reflux mechanism is not abolished, but, come as a result of acute gastric dilatation - pressing the organs within the chest cavity - or even gastric volvulus. Rarely, a hiatal hernia may present with anemia and delayed growth, as in our case. Over time, dimensions of the gap increase, the stomach is, further, attracted upwards - due to negative intra-thoracic and positive intra-abdominal pressure - the gastroesophageal junction moves in a higher position, resulting in the abolition of the anti-reflux mechanism $(1,15)$. Yazici $M$ et al.
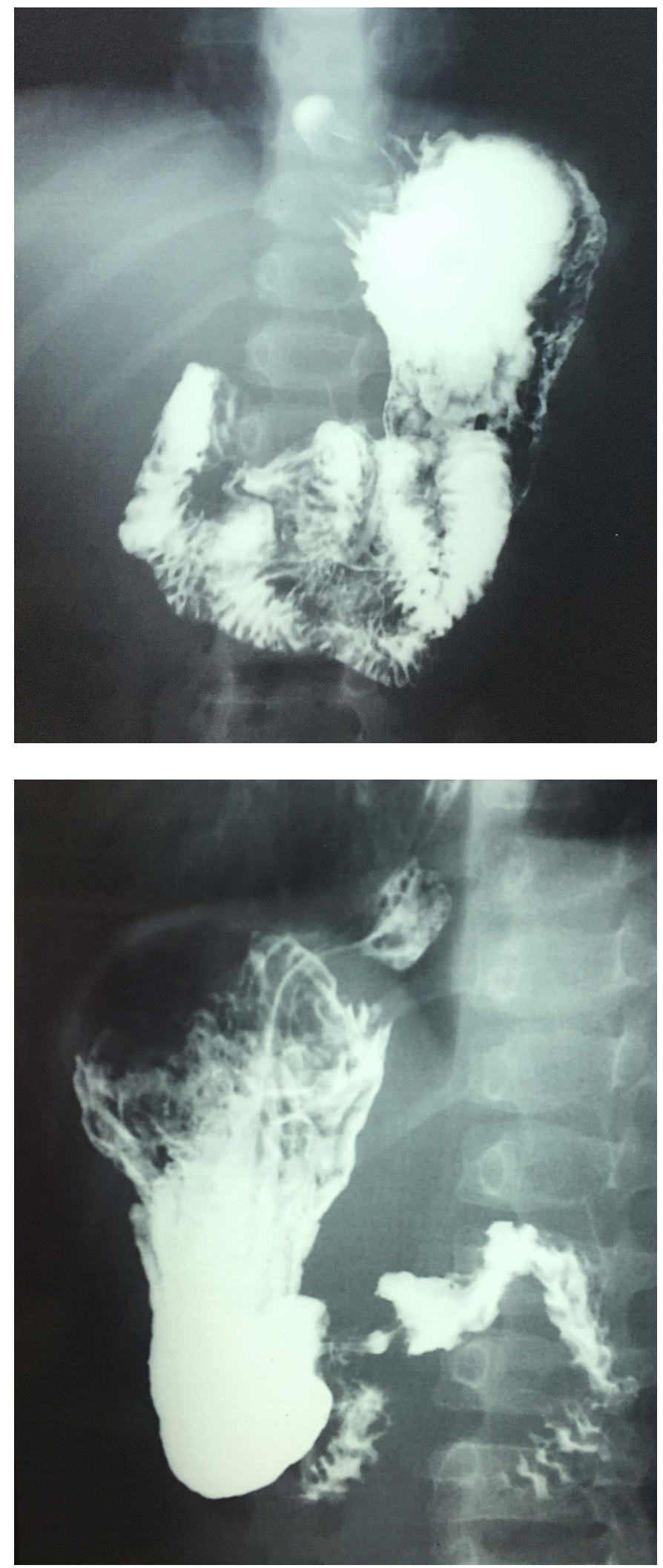

Fig. 6, 7: Barium meal examination confirmed the intra-abdominal location of the stomach, the acute gastroesophageal angle, no gastroesophageal reflux and a normal gastric emptying.

noted that $68.4 \%$ of their patients with type II hiatal hernia were suffering from gastroesophageal reflux disease (16). Enlargement of the hiatus may result in a change from type II to type IV hiatus hernia, as other intra-abdominal organs may prolapse in the chest cavity, such as epiploon, small and large intestine $(15,17,18)$. 
According to Karpelowsky JS et al., from the 5 out of 59 cases, in which type II hiatal hernia was incidentally diagnosed, in 3 out of 59 (5.01\%) patients featured subclinical anemia, later noticed, as in our case (1). Iron deficiency anemia may come as a result of slow, chronic blood loss, caused either by esophagitis - due to gastroesophageal reflux disease - or ulcers developing in the mucosa of the prolapsing stomach's part (1). In the present case, endoscopy of the upper gastrointestinal tract was performed to exclude the presence of esophagitis.

We believe that esophageal hiatal hernia, in general, along with its consequences should be included within diagnostic investigation of a child with chronic non-hereditary anemia and weight stagnation, in order to avoid a delayed diagnosis and prevent possible complications.

The diagnosis of asymptomatic type II hiatal hernia may first be suspected because of an abnormal chest x-ray, showing the gastric bubble within the thorax. In $32 \%$ of all cases, air fluid levels in the lower quadrant of mediastinum, may, also, be detected (1). Given that the hernia is asymptomatic in an early stage, a supine chest $\mathrm{x}$-ray after the placement of a nasogastric tube and repetition of it, in order to confirm the intra-thoracic place of the tube, would significantly contribute to the diagnosis $(19,20)$. The differential diagnosis includes retrosternal Morgagni hernias, mediastinal cysts, tumors and cystic echinococcosis $(1,21)$.

Barium meal examination and endoscopy of the upper digestive tract highly contribute to the confirmation of the diagnosis. Since barium meal examination showed the intra-thoracic location of the gastroesophageal junction and no findings suggestive of gastroesophageal reflux were noted after endoscopy of the upper digestive tract, the possible diagnosis of type I or III hiatal hernia was excluded. It should be pointed out that the barium meal examination is not a foolproof way to evaluate the location of the gastroesophageal junction $(1,22)$. Chest and upper abdominal MRI contributed to a more accurate evaluation of the prolapsing part, the location of the gastroesophageal junction, the width of the gap, while a possible pathology of the lower respiratory system or the mediastinum was excluded (21).

Theoretically, in type I and III hiatal hernias there is a great risk of incarceration of the prolapsing stomach, or even gastric volvulus. As a result, an elective, either laparoscopic or open, hernia repair is indicated (20). Karpelowsky JS et al. do not share with this "scaremongering" (1). During the treatment of 59 type II hiatal hernia cases, over 40 years, no such complication was noticed (1).

There is no age limit for laparoscopic hernia repair. Kundal AK et al. performed a laparoscopic hiatal hernia repair in 4 infants (20). Van der Zee DC et al. performed the same operation in 2 infants, managing, successfully, to reduce the stomach, converge the gap and perform a total fundoplication (23).

The surgical procedure of type II hernia repair includes the excision of the hernia sac, the reduction of the prolapsing stomach and the convergence of the crura of diaphragm $(9,18,24)$. It is widely believed that the excision of the hernia sac allows safer convergence of the crura of diaphragm, eliminating the risk of relapse (25). Some researchers disagree, because of possible iatrogenic injuries during hernia sac excision, suggesting that a partial excision is enough (26).

The operation is completed with anti-reflux surgerymainly, fundoplication- as performed in our patient $(1,18$, $19,21,24)$. Fundoplication is indicated in cases, in which gastroesophageal reflux disease is pre-operatively diagnosed $(1,16)$. Yousef $Y$ et al. encountered 14 little patients with type II hiatal hernia, 4 laparoscopically and 10 openly. On 13/14 patients (93\%) they performed anti-reflux surgery (22). Karpelowsky JS et al. encountered 20/59 patients, with no signs of gastroesophageal reflux disease, not performing an anti-reflux surgery (1). However, within post-operative follow up, they found that $12 / 20$ patients $(60 \%)$ developed gastroesophageal reflux disease.

It is believed that this progress is iatrogenic, due to the mobilization of the esophagus and the abolition of normal anti-reflux mechanism. With the performance of fundoplication, the stomach is stabilized in the abdomen, while a sufficient sub-diaphragmatic abdominal part of esophagus is retained (1). The operation is, preferably, performed with abdominal access, except for the cases of short esophagus, in which thoracic access is preferable (19).

Esophageal hiatal hernias with wide gap are characterized by inadequate diaphragm development, causing technical difficulty in the convergence of the gap, which is performed under tension, increasing the risk of relapse. Wang $G$ et al. suggest that the primary repair of a wide gap is technically feasible, without using prosthesis. Besides, they characterize the existence of prosthesis in a developing organism as a disadvantage (19). Another important parameter of relapse is a short esophagus, which results in a technically difficult performance of fundoplication. In this cases, Collis-Nissen procedure is preferred (27).

\section{CONCLUSIONS}

1. Esophageal hiatal hernia should be included within diagnostic approach of a child with chronic non-hereditary anemia.

2. After the diagnosis of type II esophageal hiatal hernia is established, performance of a hernia repair surgery is indicated in short time, due to the severity of possible complications.

3. Through the performance of total fundoplication, it is secured that the subdiaphragmatic abdominal part of esophagus will be retained, preventing the development of post-operative gastroesophageal reflux disease.

\section{CONFLICT OF INTEREST}

None of the contributing authors have any conflict of interest, including specific financial interests or relationships and affiliations relevant to the subject matter or materials discussed in the manuscript.

\section{REFERENCES}

1. Karpelowsky JS, Wieselthaler N, Rode H. Primary paraesophageal hernia in children. J Pediatr Surg 2006; 41(9): 1588-1593. 
2. Samiullah Ishtiaq $S$, Taimur M. Strangulated paraesophageal hiatus hernia: a case report. Isra Med J 2015; 7(3): 171-174.

3. Au Yeung KJ, Cannon ML, Arkachaisri T, et al. impact of hiatal hernia on pediatric gastroesophageal reflux disease. J Gastrointest Dig Sys 2015; 5(5): 1000330.

4. Gonzalez de Santader R. Contribucional estudo de la morfologicadesciurollo de la bursa omentalis en embrioneshumanos. An Desarrolo 1962; 10: 269-298.

5. Botha GSM. The gastroesophageal region in infants. Arch Dis Child 1958; 33: 78-94.

6. Anderson KD. Congenital diaphragmatic hernia. In: Welch KJ, Randolph JG, Ravitch MM, Rowe MC, eds. Pediatric Surgery. 4th edn. Chicago: Year Book Medical Publishers, 1986, pp. 599-606.

7. Baglaj SM, Noblett HR. Paraesophageal hernia in children: familial occurrence and review of literature. Pediatr Surg Int 1999; 15: 85-87.

8. Al-Salem AH. Congenital paraesophageal hernia with intrathoracic gastric volvulus in two sisters. Int Scholarly Research Network ISRN Surg Volume 2011, article ID 856568, 5 pages.

9. Khanna A, Finch G. Paraoesophageal herniation: a review. Surgeon 2011; 9(2): 104-111.

10. Parida SK, Kriss VM, Hall BD. Hiatus/paraesophageal hernias in neonatal Marfan's syndrome. Am J Med Genet 1997; 72(2): 156-158.

11. Jawad AJ, Al-Samarrai AI, Al-Mofada S, et al. Congenital paraesophageal hiatal hernia in infancy. Pediatr Surg Int 1998; 13(2-3): 91-94

12. Di Francesco S, Lanna M, Napolitano M, et al. A Case of Ultrasound Diagnosis of Fetal Hiatal Hernia in Late Third Trimester of Pregnancy. Case Reports in Obstetrics and Gynecology Volume 2015, Article ID 194090, 3 pages.

13. Ogunyemi. Serialsonographic findings in a fetus with congenital hiatal hernia, Ultrasound in Obstetr Gynecol 2001; 17(4):350-353.

14. Yamamoto N, Hidaka N, Anami A, et al. Prenatal sonographic diagnosis of a hiatal hernia in a fetus with asplenia syndrome. J Ultrasound Med 2007; 26(9): 1257-1261.

15. Geha AS, Massad MG, Snow NJ, Baue AE. A 32 year experience in 100 patients with giant paraesophageal hernia: The case for abdominal approach and selective antireflux repair. Surgery 2000; 128: 623630.

16. Yazici M, Karaca I, Etensel B, et al. paraesophageal hiatal hernias in children. Dis Esophagus 2003; 16(3): 210-213.

17. Al-Salem AH. Intrathoracic gastric volvulus in infancy. Pediatr Radiol 2000; 30: 842-5.

18. Mutabagani KH, Teich S, Long FR. Primary intrathoracic gastric volvulus in a new born. J Pediatr Surg 1999; 34: 1869-1871.

19. Wang G, Yang Q, Henan ZZ. Analysis of 15 cases of oesophageal hiatus hernia in children. 23rd International Congress of Paediatrics, Beijing, China, September 2001.

20. Kundal AK, Zargar NU, Krishna A. Laparoscopic repair of paraesophageal hiatus hernia in infancy Journal of Indian Association of Pediatric Surgeons 2008; 13(4): 142-143.

21. Raghunath B, DipaliParas R, Kothari Dinesh K, et al.: Congenital paraesophageal hernia presenting with severe gastroesophageal reflux. Indian J Pediatr 2007; 74(3): 310-311.

22. Yagi M, Nose K, Yamauchi K, et al. Laparoscopic intervention for intrathoracic stomach in infants. Surg Endosc 2003; 17: 1636-1639.

23. Yousef Y, Lemoine C, St-Vil D, Emil S. Congenital paraesophageal hernia: The Montreal experience. J Pediatr Surg 2015; 50(9): 1462-1466.

24. van der Zee DC, Bax NM, Kramer WL, Mokhaberi B, Ure BM. Laparoscopic management of a paraesophageal hernia with intrathoracic stomach in infants. Eur J Pediatr Surg 2001; 11: 52-54.

25. Imamoğlu M, Cay A, Kosucu P, et al. Congenital paraesophageal hiatal hernia: pitfalls in the diagnosis and treatment. J Pediatr Surg 2005; 40(7): 1128-1133.

26. Watson DI, Davies N, Devitt PG. Importance of dissection of the hernial sac in laparoscopic surgery for large hiatal hernias. Arch Surg 1999; 134: 1069-1073.

27. Percivale A, Stella M, Durante V, et al. Laparoscopic treatment of Morgagni-Larrey hernia: Technical details and report of a series. J Laparoendosc Adv Surg Tech A 2005; 15: 303-307.

28. Hu Tingze, Zhong Li, Long Shimin, et al. Evaluation of Nissen's fundoplication for gastroesophageal reflux in children. Chin J Pediatr Surg 1991; 12: 80. 